\title{
Optimal power flow with transmission cost solution under bilateral and multilateral transactions
}

\author{
Bastin Solai NaZARan J. ${ }^{1}$, K. SElvi ${ }^{2}$ \\ ${ }^{1}$ Department of Electrical and Electronics Engineering \\ Syed Ammal Engineering College, Ramanathapuram, Tamilnadu \\ India-623502 \\ e-mail:basnazar@yahoo.co.in \\ ${ }^{2}$ Department of Electrical and Electronics Engineering \\ Thiagarajar college of Engineering, Madurai \\ Tamilnadu, India-625015
}

(Received: 02.06.2013, revised: 24.03.2014)

\begin{abstract}
In a deregulated electricity market, it is important to dispatch the generation in an economical manner and to ensure security under different operating conditions. In this study evolutionary computation based solution for optimal power flow is attempted. Social welfare optimization is taken as the objective function, which includes generation cost, transmission cost and consumer benefit function. Transmission cost is calculated using Bialek's power flow tracing method. Severity index is applied as a constraint to measure the security. The objective function is calculated for pre and post contingency periods. Real power generations, real power loads and transformer tap settings are selected as control variables. Different bilateral and multilateral conditions are considered for analysis. A Human Group Optimization algorithm is used to find the solution of the problem. The IEEE 30 bus system is taken as a test system.
\end{abstract}

Key words: SEOPF, ESCOPF, power flow tracing, severity index, HGO, bilateral, multilateral transactions

\section{Introduction}

Optimal power flow is developed to solve economic dispatch with voltage and power flow constraints [1]. Later it extends to other objective functions such as loss minimization etc. A wide range of conventional optimization tools are used to solve optimal power flow problem (OPF). Nonlinear programming, Quadratic programming, Sequential quadratic programming, interior point methods and Newton based technique are the conventional techniques normally used to solve the OPF problem. But these methods suffer from insecure convergence. Due to inappropriate initial conditions sometimes the Newton method fails to converge. Computation time requirement greatly increases with the size of the problem and number of constraint violations. 
In the competitive electricity market OPF can be used to solve the inter-utility power exchange [2]. Congestion management is a major issue in the power industry. Pool prices are volatile in nature as they are spot prices. Some buyers those who do not wish to adjust their consumption may go for fixed price contracts. This concept leads to bilateral and multilateral transactions. Transaction involving one buyer-seller pair is known as bilateral transaction. Multiples of buyers and sellers grouped themselves together and enter into a transaction is known as Multilateral transaction. Bilateral and multilateral transactions must maintain their power balances. It is required to find their impacts in the growing market environment. Power injections due to these transactions can influence transmission line loading. Power flow in the transmission line may increase or decrease depending on operating conditions, transaction, power flow directions and number of transactions considered. So utilities have to decide the location of Independent Power Producers (IPPs) and the magnitude of feasible transactions. Economic dispatch solutions using evolutionary programming under bilateral, multilateral and pool transactions have been considered in literature [3-6]. Padey et al. formulated an economic dispatch problem with independent power producers [3]. IEEE 30 bus test system with continuous and discontinuous cost coefficients are considered in their paper.

Power system security is growing importance in the electricity deregulation scenario. To maintain the system security possible outages are to be studied. The N-1 criterion is used to ensure security. The contingency ranking method is used in this paper to determine credible contingencies [7].

Alleviation of transmission line overloads can be done by rerouting power flows in the network. Power flows can be modified by changing phase angles and magnitudes of bus voltages. A Fuzzy based approach has been proposed for overload alleviation through generation rescheduling in [8-9]. A Generation shift sensitivity factor was used to determine the change in generation. Although these methods are fast enough, it may lead to overloading of other unaffected lines. A GA based OPF with phase shifter for overload alleviation has been proposed in [10].

In the restructured electricity market, the transmission system is considered as an independent company that provides open access to all participants. Fair allocation of transmission cost becomes important. Different methods are practiced to allocate transmission cost worldwide. Power flow tracing method is used to allocate transmission charges in fair manner [11-15]. Bialek's power flow tracing method is used in this paper to find transmission cost [12]. A social benefit objective function which includes generation cost, transmission cost and consumer benefit is tried in [15]. In that paper pre and post contingency periods are considered but security is included as a constraint.

Evolutionary computation techniques can be used to overcome the above mentioned difficulties. These techniques exhibit quick convergence characteristics for many complex problems. The various evolutionary algorithms are genetic algorithm [16], particle swarm optimization [17] and differential evolution, etc. These algorithms mimic evolving population leads to the global optimum. Even though PSO and DE perform well, they are prone to premature convergence, stagnation and choosing correct control parameters. Motivated from No Free Lunch theorem several new algorithms are also developed. Seeker Optimization Algorithm is 
proposed in [18]. It is a new population based heuristic algorithm which simulates the performance of human searching in real parameter optimization. The authors now renamed the algorithm as Human Group Optimization [18-23].

The objective function detailed in [24] with simplified assumptions is used in this paper. Normal optimal power flow problem is modified to include expected security costs. The objective function includes generation cost, transmission cost and consumer benefit function for the base case and post contingency cases. The need to include the post contingency period cost is to ensure feasible solution in post contingency periods. Load interruption cost is not included in this paper. The simplified ESCOPF objective function proposed is solved for different transactions using HGO. Results are compared with PSO and DE.

\section{Problem formulation}

The objective of the problem is to minimize the generation and transmission cost under bilateral and multilateral transaction condition with security enhancement. In [15] consumer benefit function is used along with generation and transmission cost but pool type transaction are only tried. In [24] generation cost, security costs and consumer benefit function are addressed but transmission cost is not included. This problem is stated as ESCOPF, i.e. Expected security cost OPF.

\subsection{Objective function}

The main objective of this paper is to minimize the expected overall cost of the system while satisfying set of equality and inequality constraints. Minimization of expected overall cost includes pre contingency period and post contingency period. The objective function addressed incorporates expected security cost along with generation and transmission cost. By including all credible contingency cost into the objective, feasible solution is ensured during post contingency period. Minimization of expected overall cost includes pre contingency period and post contingency period. Post contingency load interruption is not included in the objective function.

$$
\begin{gathered}
F=\min \left\{C_{0}+\sum_{k=1}^{K} C_{k}\right\}, \\
C=\sum_{i=1}^{n_{g}} f\left(P_{G i}\right)+\sum_{i=1}^{n_{g}} T C_{i}-\sum_{i=1}^{n_{d}} B\left(P_{D i}\right),
\end{gathered}
$$

where $F$ is objective function, $K$ is total no. of credible contingencies. $n_{g}$ is total number of available generators, $n_{d}$ is total number of loads, $C$ is the total cost of generation and transmission for the specified condition $\$ / \mathrm{hr}, C_{0}$ is base case (pre contingency) cost, $C_{k}$ is post contingency cost of $k^{\text {th }}$ contingency, $P_{G i}$ is active power supplied by $i^{\text {th }}$ generator, $f\left(P_{G i}\right)$ is generation cost function $\$ / \mathrm{hr}, T C$ represents transmission cost $\$ / \mathrm{hr}, B\left(P_{D i}\right)$ represents consumer benefit function. 


$$
T C_{i}=\sum_{m=1}^{n_{\text {inese }}} c_{m}\left(M W_{G i, m}\right) l_{m} M W_{G i, m},
$$

where $T C_{i}$ transmission cost due to $i^{\text {th }}$ generator, $c_{m}$ cost per MW per unit length of line $m \$ / \mathrm{hr}, l_{m}$ length of line $\mathrm{m}$ in miles, $M W_{G i, m}$ real power flow in line $m$ due to $i^{\text {th }}$ generator, $n_{\text {lines }}$ number of lines get contribution from $i^{\text {th }}$ generator.

The objective function (1) is a modified version of the objective function proposed in [24] with a security constraint.

\subsection{Constraints}

Equality constraints

This is nonlinear steady state power balance equations

$$
\begin{gathered}
P_{G i}-P_{D i}-\sum_{j=1}^{n_{b u s}}\left|V_{i}\right|\left|V_{j}\right|\left|Y_{i j}\right| \cos \left(\theta_{i j}-\delta_{i}+\delta_{j}\right)=0, \\
Q_{G i}-Q_{D i}+\sum_{j=1}^{n_{b u s}}\left|V_{i}\right|\left|V_{j}\right|\left|Y_{i j}\right| \sin \left(\theta_{i j}-\delta_{i}+\delta_{j}\right)=0,
\end{gathered}
$$

where $P_{G i}$ is active power generation at bus $i ; P_{G i}$ is active power demand at bus i; $Q_{G i}$ is reactive power generation at bus $i$; $Q_{D i}$ is reactive power demand at bus $i ;\left|V_{i}\right|$ voltage magnitude at bus $i ;\left|Y_{i j}\right|$ is magnitude of bus admittance; $\theta_{i j}$ is angle of bus admittance; $\delta_{i}$ is voltage angle and $n_{\text {bus }}$ is number of buses in the system.

Inequality constraints

Active power Generation of each unit must be within their upper and lower limits

$$
P_{G i_{-} \min } \leq P_{G i} \leq P_{G i_{-} \max } .
$$

The voltage at load buses must be within their limits

$$
V_{j_{-} \min } \leq V_{j} \leq V_{j_{-} \max } .
$$

\section{Security Constraint}

In this paper Severity Index [7] is added as a constraint to be satisfied. For each credible contingency the expected value is zero.

Severity Index

$$
S I_{k}=\frac{1}{2^{m}} \sum_{l \in L_{0}}\left(\frac{S_{l}}{S_{l}^{\max }}\right)^{2 m},
$$

where $S I_{k}=$ Severity Index of $k^{\text {th }}$ line outage, $S_{l}^{\max }=$ MVA rating of line $l ; S_{l}=$ Power flow in line $l$ (MVA); $L_{0}=$ Set of overloaded lines; $m=1$.

This constraint ensures overload alleviation of transmission lines. 


\section{Human Group Optimization}

The HGO (Human Group Optimization) is a population-based heuristic search algorithm. It regards the optimization process as an optimal solution obtained by a search population and each individual of this population is called a seeker (A person). The total population is randomly categorized into three subpopulations. These subpopulations search over several different domains of the search space. All the seekers in the same subpopulation constitute a neighbourhood. This neighbourhood represents the social component for the social sharing of information.

Steps of Human Group Optimization:

In the HGO, a search direction $d_{i j}(t)$ and a step length $\alpha_{i j}(t)$ are to be computed separately for each $i^{\text {th }}$ seeker on each $j^{\text {th }}$ variable at each time step $t$, where $\alpha_{i j}(t) \geq 0$ and $d_{i j}(t) \in\{-1,0,1\}$ where, $i$ represents the population number and $j$ represents the optimizing variable number.

a) Calculation of the search direction, $d_{i j}(t)$ : Swarm exhibits two extreme types of cooperative behaviour. One is entirely pro-self based (egotistic) and another is entirely pro-group based (altruistic). Every seeker believes that he should go toward his historical best position $\vec{P}_{i, b e s t}(t)$ according to his own judgment. This attitude of $\mathrm{i}^{\text {th }}$ seeker may be modelled by an empirical direction vector $\vec{d}_{i, e g o}(t)$ as shown in (9).

$$
\vec{d}_{i, \text { ego }}(t)=\operatorname{sign}\left(\vec{p}_{i, \text { best }}(t)-x_{i}(t)\right) .
$$

Here sign $(\cdot)$ is a Signum function on each dimension of the input vector. Whereas, based on the altruistic behaviour seekers in the same neighbourhood cooperate explicitly to achieve desired goal. The population exhibits a self organised aggregation behaviour in the form of attraction toward a given signal source. For a black box problem in which global minimum value is unknown, historical best position $\vec{g}_{\text {best }}(t)$ of neighbourhood or neighbours' current best position $\vec{l}_{\text {best }}(t)$ is used as an attraction signal source of the self-aggregation behaviour. Each seeker is associated with two altruistic directions $\vec{d}_{i, a l t 1}(t)$ and $\vec{d}_{i, a l t 2}(t)$ as follows

$$
\begin{gathered}
\vec{d}_{i, a l t 1}(t)=\operatorname{sign}\left(\vec{g}_{\text {best }}(t)-\vec{x}_{i}(t)\right), \\
\vec{d}_{i, \text { alt } 2}(t)=\operatorname{sign}\left(\vec{l}_{\text {best }}(t)-\vec{x}_{i}(t)\right),
\end{gathered}
$$

In addition to that seekers exhibit proactive behaviour i.e., future behaviour can be predicted and guided by past behaviour. Hence each seeker associated with a search direction $\vec{d}_{i, p r o}(t)$ as follows

$$
\vec{d}_{i, p r o}(t)=\operatorname{sign}\left(\vec{x}_{i}\left(t_{1}\right)-\vec{x}_{i}\left(t_{2}\right)\right),
$$

Where $t_{1}, t_{2} \in\{t, t-1, t-2\}$ and $\vec{x}_{i}\left(t_{1}\right)$ is better than $\vec{x}_{i}\left(t_{2}\right)$.

Based on human rational judgement, the actual direction is selected as compromise among the aforementioned directions. A proportional selection rule is used to find the direction $d_{i j}$.

Let $m \in\{0,+1,-1\}$ and $p_{j}^{(m)}=($ The number of $m) / 4$ then 


$$
d_{i j}=\left\{\begin{array}{l}
0, \quad \text { if } r_{j} \leq p_{j}^{(0)} \\
+1, \text { if } \quad p_{j}^{(0)} \leq r_{j} \leq p_{j}^{(0)}+p_{j}^{(+1)} \\
-1, \quad \text { if } p_{j}^{(0)}+p_{j}^{(+1)}<r_{j} \leq 1
\end{array}\right.
$$

Here $r_{j}$ is a uniform random number between 0 and 1 .

b) Calculation of the step length $\alpha_{i j}(t)$ : Human searching behaviour can be modelled with fuzzy reasoning. One may find the near optimal solution in the narrower neighbourhood of lower fitness value and wider neighbourhood of higher fitness value. As different optimization problems have different ranges of fitness values, it is required to design a fuzzy system to be applicable to a wide range of optimization problems. So the fitness values of all the seekers are sorted in descending manner (for minimization problem) and turned into the sequence numbers from 1 to $S$ as the inputs of fuzzy reasoning. The linear membership function is used

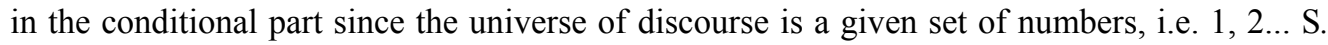
The expression is presented as in (14).

$$
\mu_{i}=\mu_{\max }-\frac{S-I_{i}}{S-1}\left(\mu_{\max }-\mu_{\min }\right),
$$

Where $\mu_{\max }$ and $\mu_{\min }$ are maximum and minimum membership values respectively. $I_{i}$ is sequence number obtained after sorting of $\vec{x}_{i}(t)$.

A Bell membership function

$$
\mu(x)=e^{-x^{2 / 2 \delta^{2}}}
$$

is used as action part. Membership values beyond $\pm 3 \delta(\mu( \pm 3 \delta)=0.0111)$ are less than 0.0111 . So that can be neglected. The action part of the fuzzy reasoning is shown in Figure 1.

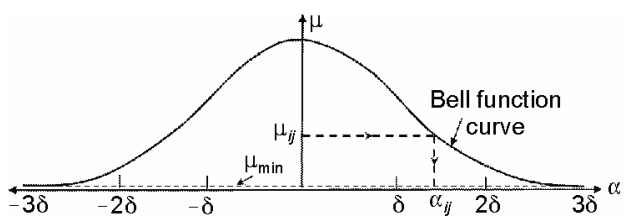

Fig. 1. The action part of the Fuzzy reasoning

The parameter $\delta$ of the membership function is determined by the following equation:

$$
\vec{\delta}=\omega \cdot a b s\left(\vec{x}_{\text {best }}-\vec{x}_{\text {rand }}\right)
$$

In order to improve the search precision a parameter $\omega$ is used. $\omega$ is linearly varied from 0.9 to 0.1 during a run. $\vec{x}_{b e s t}$ and $\vec{x}_{\text {rand }}$ are selected from same subpopulation and they are different seekers. $\vec{\delta}$ is shared by all seekers in the same subpopulation.

In order to introduce randomness in each variable and to improve searching ability the following equation is used.

$$
\mu_{i j}=R A N D\left(\mu_{i}, 1\right)
$$


In Equation (16) a uniform random real number within $\left[\mu_{i}, 1\right]$ is returned. The step length of each variable $j$ is given by

$$
\alpha_{i j}=\delta_{j} \sqrt{-\ln \left(\mu_{i j}\right)} .
$$

c) Updating seekers' position: The position update of $i^{\text {th }}$ seeker on the $j^{\text {th }}$ dimension is given by

$$
x_{i j}(t+1)=x_{i j}(t)+\alpha_{i j}(t) d_{i j}(t) .
$$

d) Binomial crossover: Since subpopulations are gathering information within its own domain, there is a possibility of local minima. In order to avoid this worst K-1 seekers are combined with the best of other subpopulations using the following binomial crossover:

$$
x_{k_{n} j, \text { worst }}=\left\{\begin{array}{ccc}
x_{l j, \text { best }} & \text { If } & R_{j \leq 0.5} \\
x_{k_{n} j, \text { worst }}, & \text { else }
\end{array}\right.
$$

Where $R_{j}$ is uniformly distributed random number within $[0,1] . x_{k_{n} j \text {,worst }}$ is $j^{\text {th }}$ dimension of a $n^{\text {th }}$ worst position in the $k^{\text {th }}$ subpopulation. $x_{l j, b e s t}$ is $j^{\text {th }}$ dimension of best position in the $l^{\text {th }}$ subpopulation with $n, k, l=1,2, \ldots, K-1$ and $k \neq l$. Good information acquired so for is shared among subpopulations using binomial crossover.

\section{Transmission cost allocation}

Allocation of transmission cost is crucial in power system studies under deregulated environment. It is expected that the transmission cost allocation scheme must be very fair, simple and accurate and also that can recover total cost. There are several methods available to allocate transmission cost. Most popular methods are postage stamp method, contract path method, MW-Mile method and power flow tracing method [11]. Most of the methods are inaccurate methods and not providing enough revenues. In these methods power flow tracing method is accurate and simple algorithm to understand. It is an actual power flow based method. In this paper Bialek's method of power flow tracing [12] is used to find the share of generation to line flow.

The proportional sharing principle is used to determine the contributors to network usage. It is assumed that Bus is a perfect mixture of all incoming flows. Power in the outgoing lines is decomposed into $\mathrm{M}$ shares, where $\mathrm{M}$ is the number of incoming flows. The amount of such shares has the same proportion as that of incoming flows to the Bus. Figure 2 portrays the proportional sharing principle.

Bialek's approach uses matrix manipulations to find topological distribution factors. The generation distribution factors able to calculate the share of generators to line flow. IPPs can get increase in profit through a lessening of power-transfer payments and/or a modification of the pool/bilateral mix. Consumers can reduce expenditures through a lessening of powertransfer payments and/or a modification of the pool/bilateral mix. 


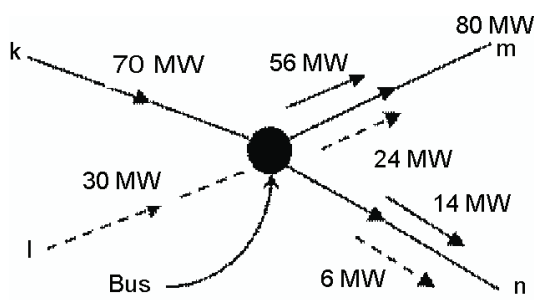

Fig. 2. Proportional sharing Principle

So by using pool/bilateral mix generation cost, transmission cost and consumer benefit are improved. There are two versions in Bialek's power flow tracing: Upstream algorithm and downstream algorithm. Upstream algorithm allocates transmission usage charge to individual generators. So it is used in this paper. Gross power flow through transmission lines and gross power injected to a node is considered in upstream algorithm. The gross power balance equation at node $i$ may be expressed, when looking at the inflows, as

$$
P_{i}^{\text {gross }}=\sum_{j \in \alpha_{i}^{u}}\left|P_{i-j}^{\text {gross }}\right|+P_{G i} \quad \text { for } i=1,2, \ldots, n,
$$

where $\alpha_{i}^{u}$ is the set of nodes supplying directly node $i$ (i.e. power must flow towards node $i$ in the relevant lines), $P_{i-j}^{\text {gross }}$ is the line flow (gross) into node $i$ in line $j-i$, and $P_{G i}$ is the generation at node $i$. As the losses are broken and added to respective nodal demands, $\left|P_{j-i}^{\text {gross }}\right|=\left|P_{i-j}^{\text {gross }}\right|$.

The line flow can be related to nodal flow at node $j$ by substituting $\left|P_{i-j}^{\text {gross }}\right|=c_{j i}^{\text {gross }} P_{j}^{\text {gross }}$, where $c_{j i}^{\text {gross }}=\left|P_{j-i}^{\text {gross }}\right| / P_{j}^{\text {gross }}$ and as losses are small this expression can be written as $c_{j i}=P_{j-i}$ $/ P_{j}$. Equation (20) can be rewritten as

$$
P_{i}^{\text {gross }}-\sum_{j \in \alpha_{i}^{u}} c_{i i} P_{j}^{\text {gross }}=P_{G i} \quad \text { or } A_{u} P^{\text {gross }}=P_{G},
$$

Here, $A_{u}$ is the $(n \times n)$ upstream distribution matrix. $P^{g r o s s}$ is the unknown vector of nodal through-gross flows and $P_{G}$ is the vector of nodal generations. The $(i, j)$ element of $A_{u}$ is equal to

$$
\left[A_{u}\right]_{i j}=\left\{\begin{array}{ccc}
1 & \text { for } & i=j \\
-c_{j i} & \text { for } & j \in \alpha_{i}^{u} . \\
0 & \text { otherwise }
\end{array}\right.
$$

Note that $A_{u}$ is sparse and nonsymmetric. If $A_{u}^{-1}$ exists then $P^{\text {gross }}=A_{u}^{-1} P_{G}$ and its $i^{\text {th }}$ element is

$$
P_{i}^{\text {gross }}-\sum_{k=1}^{n}\left[A_{u}^{-1}\right] P_{G k} \quad \text { for } i=1,2, \ldots, n .
$$

This equation shows that the contribution of the $k^{\text {th }}$ system generator to $i^{\text {th }}$ nodal gross power is equal to $\left[A_{u}^{-1}\right]_{j k} P_{G k}$. 
A gross outflow in line $i-l$ from node $i$ can be therefore calculated, using the proportional sharing principle, as

$$
\left|P_{i-l}^{\text {gross }}\right|=\frac{\left|P_{i-l}^{\text {gross }}\right|}{P_{i}^{\text {gross }}} P_{i}^{\text {gross }} \approx \frac{\left|P_{i-l}\right|}{P_{i}} \sum_{k=1}^{n}\left[A_{u}^{-1}\right]_{j k} P_{G k} \quad \text { for all } l \in \alpha_{i}^{d},
$$

$\alpha_{i}^{d}$ is set of nodes directly supplied from node $i$.

By using this equation contribution of each generator to line flow can be calculated $\left(M W_{G i, m}\right)$. Once the contribution of each generator on a transmission line is known, then MW-Mile methodology of transmission pricing can be employed. The share of generators of transmission cost is calculated using Equation (3). Transmission cost is a function of power flow through transmission lines. Bialek's power flow tracing method enables to find the influence of different transaction and able to calculate the usage of particular line by a transaction. It is also possible to alleviate overload by adjusting pool generation and location of IPP's.

\section{Proposed methodology}

The proposed method to solve security enhanced OPF with transmission cost solutions for different transactions are described in steps.

Step 1. Choose the power transaction value of bilateral / multilateral transactions.

Step 2. Choose population size, no of subpopulations, $\mu_{\max }, \mu_{\min }$ values, maximum generations and control variable limits.

Step 3. Generate initial random population (seekers) and set generation count $t=0$.

Step 4. For each seeker run Newton Raphson power flow twice to find Slack bus power, power flow through branches under pre and post contingency cases (one severe contingency is considered) .

Step 5. Run power flow tracing to find the generators contribution to branch flows and calculate Transmission cost.

Step 6. Calculate fitness function.

In this paper constraints are handled by penalization. Fitness function is formed by adding penalty terms with objective function. Fitness function is represented by

Minimize

$$
F F_{i}=F_{i}+p f_{1} \times S I_{i}+p f_{2} \times V_{\text {err }, i},
$$

where $F F_{i}$ - fitness function of $i^{\text {th }}$ candidate solution; $p f_{1} \& p f_{2}$ are penalty factors, $V_{\text {err }}$ is sum of deviation of load bus voltages from their limits, $F$ - objective function (1), $S I$ - severity index (8).

Step 7. Find the personal best, neighbourhood best and population best.

Step 8. Compute search direction $d_{i j}$ and step length $\alpha_{i j}$.

Step 9. Update the position of each seeker using (18). 


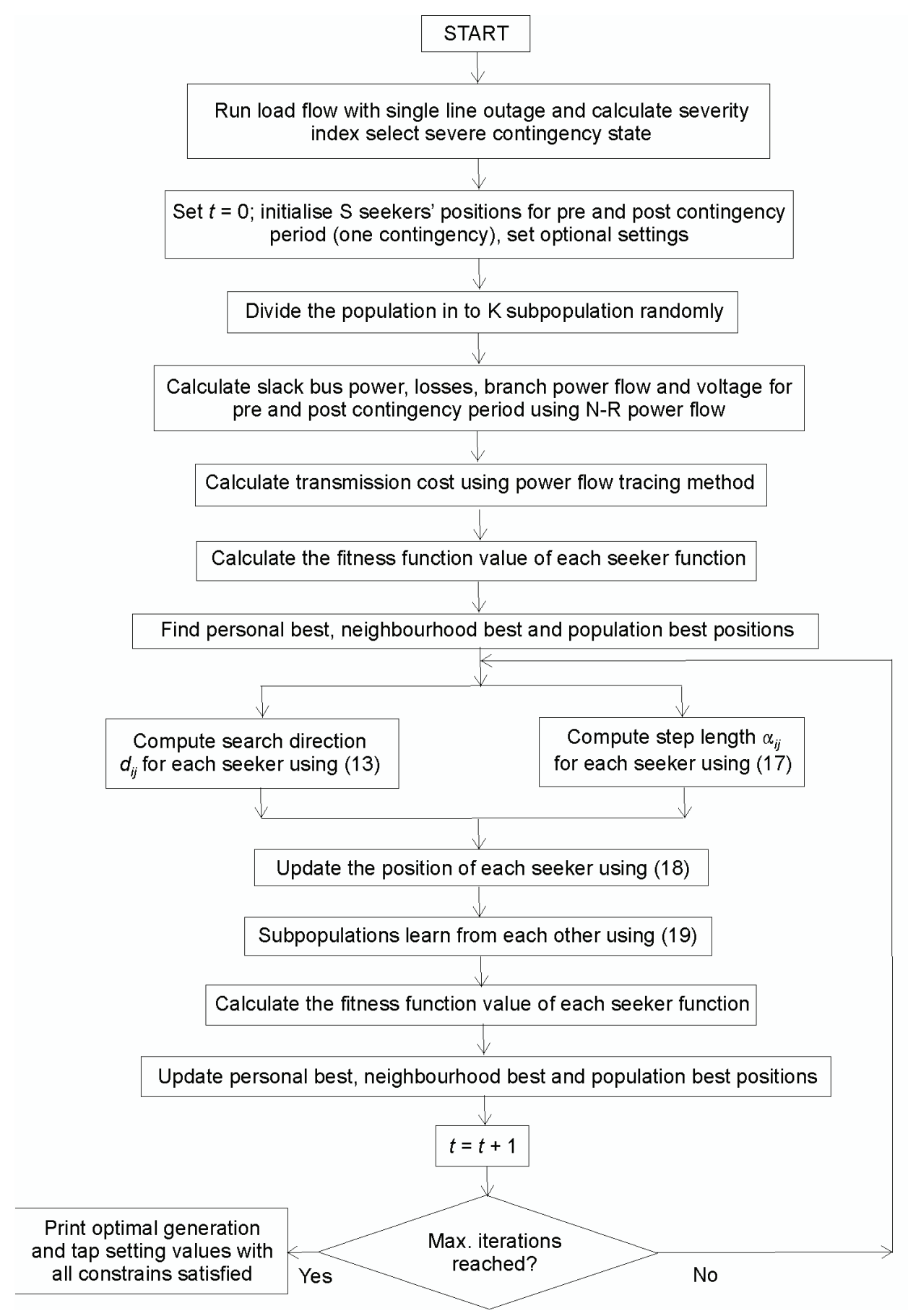

Fig. 3. Flow chart of proposed methodology 
Step 10. Let subpopulations learn from neighbourhood using (19).

Step 11. Calculate fitness function and update personal best, neighbourhood best and population best.

Step 12. Check for termination condition, print results else go to step 8 .

In the solution string shown in Figure 4, first $n$ variables are assigned to the pre contingency case and next $n$ variables are assigned to first of $k^{\text {th }}$ contingency considered. Control variables $(x)$ considered are the real power generation excluding the slack bus, PV bus voltages and tap setting of transformers. In this paper only one critical contingency is considered for analysis to reduce the computational burden. That critical contingency is computed using contingency analysis [7]. In IEEE 30 bus system line 1-2 outage is found to be critical contingency with a severity index of 5.5803. Algorithmic steps are shown well in Figure 3.

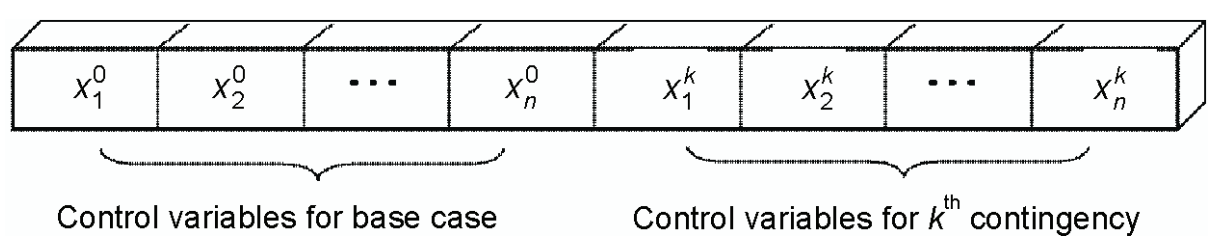

Fig. 4. Seekers' solution string

\section{Simulation results and discussion}

The proposed method is implemented under MATLAB 7.5 Environment. MATPOWER package is used for computing power flow details [25]. HGO algorithm is taken for analysis and results are compared with PSO and DE. IEEE 30 bus system is used to evaluate the methodology. The IEEE 30 bus system contains 6 generators, 4 LTC transformers and 41 transmission lines. Four transaction cases are considered. All the algorithms are run for 20 times.

Case I. Bilateral Transaction: $10 \mathrm{MW}$ of power injected at generator bus 5 and same amount is consumed from load bus 29. The voltage profile of buses is shown in Figure 5. Individual generator contribution to branch power flow (except bilateral power transacted) at base case is shown in Figure 6. Generator contribution to power flow at post contingency case is shown in Figure 7. Lline 1-2 outage is considered as critical contingency case. The convergence characteristics of different algorithms are shown in Figure 8.

Case II. Bilateral Transaction: $10 \mathrm{MW}$ of power injected at load bus 29 and same amount is consumed from generator bus 5 . The voltage profile of buses, Individual generator contribution to branch power flow at base case and Generator contribution to power flow at post contingency case are shown in Figures 9-11 respectively.

Case III. Multilateral Transaction: Injection of $10 \mathrm{MW}$ and $10 \mathrm{MW}$ in generator buses 5 and 8 respectively. Consumption of $5 \mathrm{MW}$ and $15 \mathrm{MW}$ in load buses 20 and 29 respectively. The bus voltage profile, generator contribution to power flow at base case and contingency case are shown in Figures 12,13 and 14 respectively. 
Case IV. Multilateral Transaction: Injection of $10 \mathrm{MW}$ and $10 \mathrm{MW}$ in load buses 20 and 29 respectively. Consumption of 5MW and $15 \mathrm{MW}$ in generator buses 5 and 8 respectively. Different characteristics mentioned above are shown in figures 15, 16, 17 and 18.

Table 1. HGO optimization result for IEEE 30 bus system without any of the transactions

\begin{tabular}{c|c|c|c|c|c|c|c|c|c|c|c|c|c|c|c}
\hline State & P1 & P2 & P5 & P8 & P11 & P13 & T1 & T2 & T3 & T4 & $\begin{array}{c}\text { GC } \\
\$ / h r\end{array}$ & $\begin{array}{c}\text { TC } \\
\$ / h r\end{array}$ & $\begin{array}{c}\text { CBF } \\
\$ / h r\end{array}$ & $\begin{array}{c}\text { Loss } \\
\text { MW }\end{array}$ & $\begin{array}{c}\text { Fitne } \\
\$ / h r .\end{array}$ \\
\hline PRE & 118.5 & 55.3 & 46.8 & 34.9 & 30 & 39.9 & 0.9 & 1.1 & 1.1 & 1.07 & 1018.8 & 918.4 & -5367 & 5.93 & \multirow{2}{*}{-6801.6} \\
\hline POST & 92.67 & 79.6 & 49.9 & 34.8 & 30 & 39.9 & 0.94 & 1.1 & 1.1 & 1.08 & 1068.5 & 926.7 & -5367 & 7.51 & \\
\hline
\end{tabular}

Table. 2. HGO optimization Results for IEEE 30 bus system with transactions

\begin{tabular}{l|c|c|c|c|c|c|c|c}
\hline & \multicolumn{2}{|c|}{ Case I } & \multicolumn{2}{c|}{ Case II } & \multicolumn{2}{c}{ Case III } & \multicolumn{2}{c}{ Case IV } \\
\hline & Pre & Post & Pre & Post & Pre & Post & Pre & Post \\
\hline P1 (MW) & 117.35 & 92.2 & 119 & 93.10 & 120.1 & 92.62 & 122.07 & 93.62 \\
\hline P2 (MW) & 55.49 & 79.49 & 55.86 & 79.45 & 54.02 & 79.54 & 53.64 & 79.61 \\
\hline P5 (MW) & 47.13 & 49.96 & 46.10 & 49.96 & 46.41 & 50 & 45.88 & 49.88 \\
\hline P8 (MW) & 34.97 & 34.99 & 34.94 & 34.99 & 34.97 & 34.96 & 34.96 & 34.98 \\
\hline $\begin{array}{l}\text { P11 } \\
\text { (MW) }\end{array}$ & 30 & 29.99 & 29.95 & 29.99 & 29.96 & 29.95 & 29.93 & 30 \\
\hline $\begin{array}{l}\text { P13 } \\
\text { (MW) }\end{array}$ & 40 & 39.99 & 39.99 & 40 & 40 & 39.96 & 39.96 & 39.91 \\
\hline T1 & 0.9 & 0.92 & 0.9 & 0.94 & 0.9 & 0.94 & 0.9 & 0.92 \\
\hline T2 & 1.1 & 1.09 & 1.08 & 1.09 & 1.09 & 1.1 & 1.1 & 1.1 \\
\hline T3 & 1.07 & 1.07 & 1.06 & 1.09 & 1.08 & 1.09 & 1.08 & 1.08 \\
\hline T4 & 1.06 & 1.06 & 1.05 & 1.07 & 1.07 & 1.08 & 1.07 & 1.06 \\
\hline $\begin{array}{l}\text { GCost } \\
\$ / \mathrm{hr}\end{array}$ & 1019.54 & 1068.3 & 1018.3 & 070.5 & 1017.2 & 1069.4 & 1017.4 & 1071.6 \\
\hline $\begin{array}{l}\text { Trcost } \\
\text { \$/hr }\end{array}$ & 913.43 & 922.74 & 926.2 & 932.6 & 919.0 & 925.5 & 929.93 & 934.94 \\
\hline CBF \$/hr & 5363.00 & 5363.00 & 5374.95 & 5374.95 & 5366.75 & 5366.75 & 5377.9 & 5377.9 \\
\hline $\begin{array}{l}\text { Social } \\
\text { welfare } \\
\$ / \mathrm{hr}\end{array}$ & -3430.02 & -3371.91 & -3430.46 & -3371.76 & -3430.57 & -3371.9 & -3430.6 & 3371.3 \\
\hline $\begin{array}{l}\text { Loss MW } \\
\text { Fitness }\end{array}$ & 5.725 & 7.472 & 5.838 & 7.501 & 5.935 & 7.471 & 6.027 & 7.601 \\
\hline (hr & -6801.94 & -6802.23 & -6802.47 & & -6801.96 \\
\hline
\end{tabular}


Table 3. Statistical Analysis of IEEE 30 bus system

\begin{tabular}{|c|c|c|c|c|c|c|c|c|c|c|c|c|}
\hline \multirow{2}{*}{$\begin{array}{c}\text { Algo- } \\
\text { rithm }\end{array}$} & $\begin{array}{c}\text { Best } \\
\text { fitness }\end{array}$ & $\begin{array}{c}\text { CASE I } \\
\text { fitness }\end{array}$ & $\begin{array}{c}\text { Stan- } \\
\text { dard } \\
\text { devia- } \\
\text { tion }\end{array}$ & $\begin{array}{c}\text { Best } \\
\text { fitness }\end{array}$ & $\begin{array}{c}\text { Worst } \\
\text { fitness }\end{array}$ & $\begin{array}{c}\text { Stan- } \\
\text { dard } \\
\text { devia } \\
\text { tion }\end{array}$ & $\begin{array}{c}\text { Best } \\
\text { fitness }\end{array}$ & $\begin{array}{c}\text { Worst } \\
\text { fitness }\end{array}$ & $\begin{array}{c}\text { Stan- } \\
\text { dard } \\
\text { devia- } \\
\text { tion }\end{array}$ & $\begin{array}{c}\text { Best } \\
\text { fitness }\end{array}$ & $\begin{array}{c}\text { Worst } \\
\text { fitness }\end{array}$ & $\begin{array}{c}\text { CASE III } \\
\text { devia- } \\
\text { tion }\end{array}$ \\
\hline HGO & -6801.9 & -6800.6 & 0.39 & -6802.2 & -6798.5 & 1.01 & -6802.5 & -6799.2 & 0.79 & -6802 & -6799.3 & 0.66 \\
\hline DE & -6703.4 & -6667.8 & 9.16 & -6703.7 & -6665.1 & 9.74 & -6703.5 & -6669.7 & 9.08 & -6703.2 & -6682 & 7.22 \\
\hline PSO & -6686.1 & -6600.3 & 19.27 & -6702.1 & -6556 & 36.30 & -6699.7 & -6542.4 & 31.1 & -6692.4 & -6556.1 & 31.23 \\
\hline
\end{tabular}

The optimum control values and their associated generation, transmission costs are tabulated for different cases in Table 1. Severity index for all these cases are zero. Post contingency generation and transmission cost is more compare to pre contingency case due to change in power flow. When the load is kept constant in the post contingency case then the generation (Pool) alters to alleviate overloads that causes change in transmission cost. In case III due to multilateral transaction generation and transmission cost are more compare to all other cases. Real power loss is also high in this case. From Table 2 it is possible to observe that some transactions reduce operating cost and some increase operating cost. Comparing the bilateral transactions, transaction 2 reduces the overall operating cost. Similarly comparing the multilateral transactions, transaction 1 reduces the overall operating cost. So utility can decide where to encourage IPP participation by using this method. From Table 2 transmission cost is less for transaction 4. This is due to loads are supplied locally through transactions. We can easily verify the contribution of individual generators to line flow and observe the differences through figures shown. These are drawn using the data obtained from the power flow tracing. In Figure 16 power injection at bus 20 causes power flow through line 24. Similarly, power injection at bus 29 causes power flow through line 39 alone. The transmission cost is high when power is taken from generator buses (Case II \& Case IV). As power is supplied from the pool generators, optimal path taken by the power flow varies that causes increase in transmission cost. Real power loss is also high compared to other cases.

Fig. 5. Bus voltage profile (Case I)

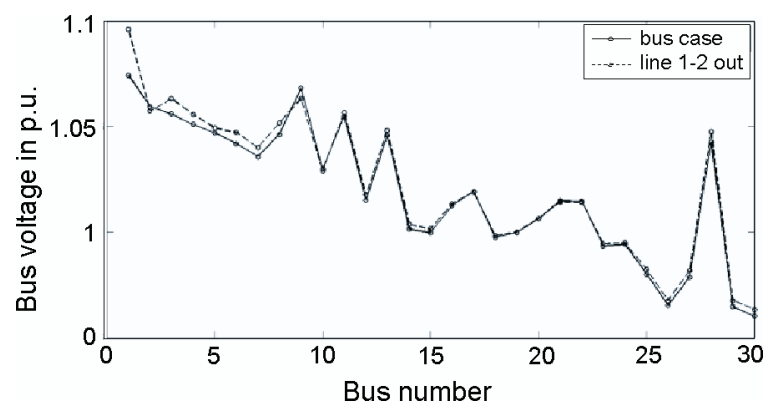

From the statistical data shown in Table 3. The best fitness values obtained for HGO and $\mathrm{DE}$ are closer. The worst values among different runs show the difference between these two algorithms in the IEEE 30 bus case. Among three algorithms HGO stands good in standard 


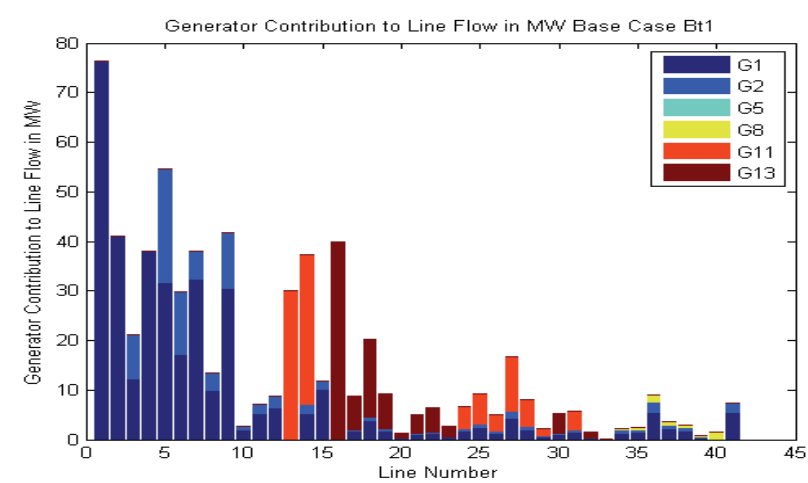

Fig. 6. Generator contribution to line flow in at base case (Case I)

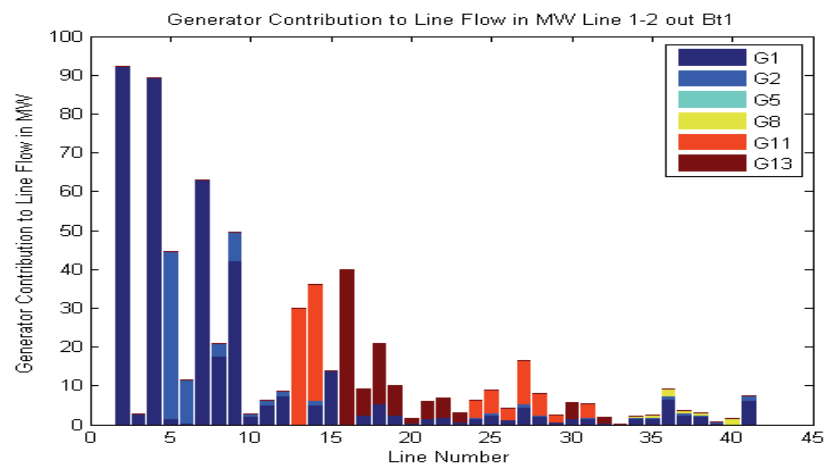

Fig. 7. Generator contribution to line flow at line 1-2 out (Case I)

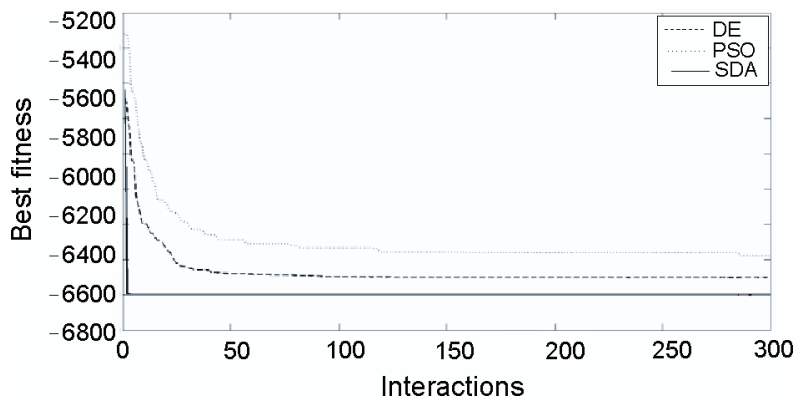

Fig. 8. Convergence characteristics (Case I)

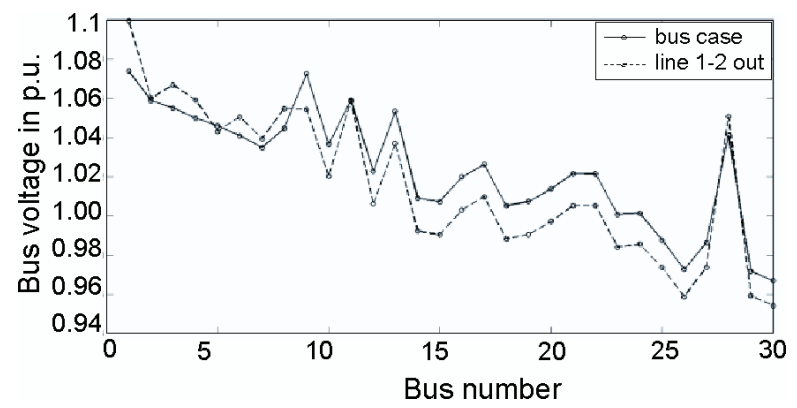

Fig. 9. Bus voltage profile (Case II) 
Fig. 10. Generators contribution to line flow at base case (Case II)

Fig.11. Generators contribution to line flow Line 1-2 out (Case II)
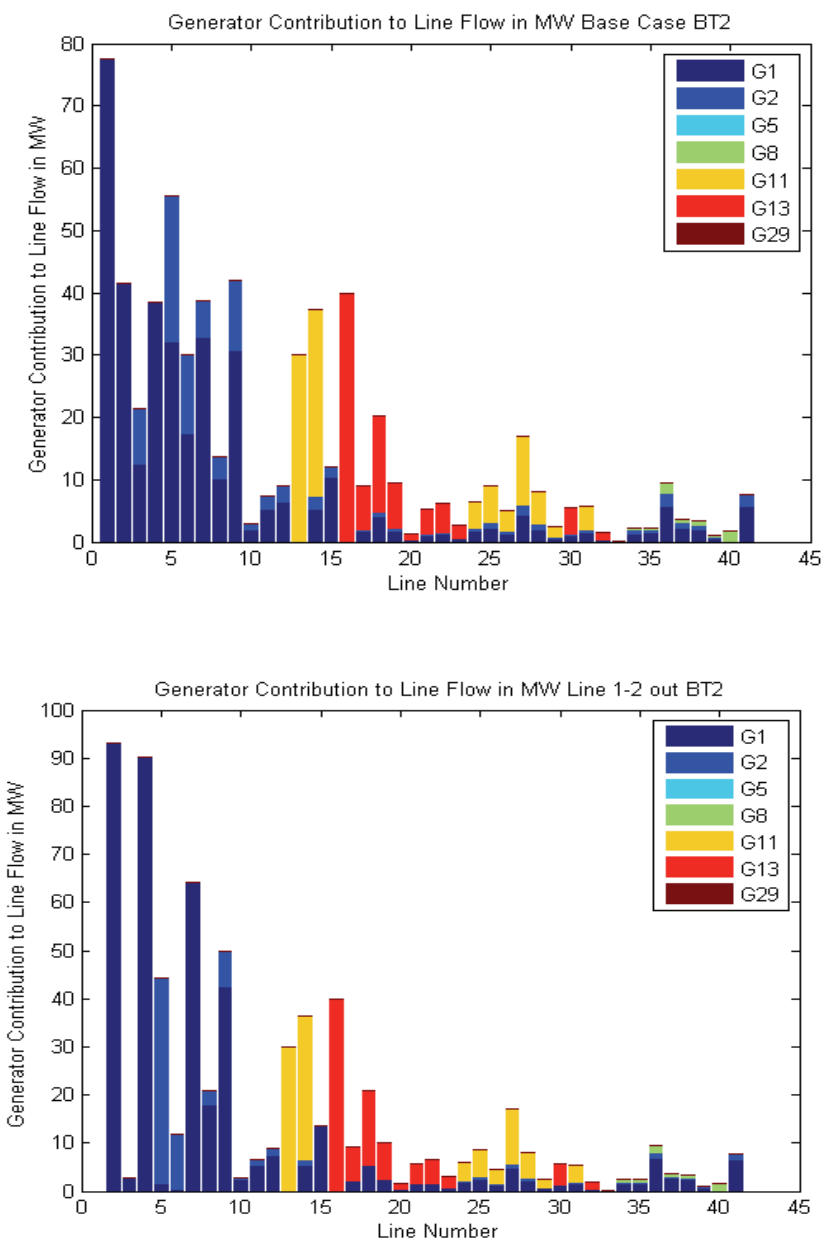

Fig. 12. Bus Voltage profile Case III

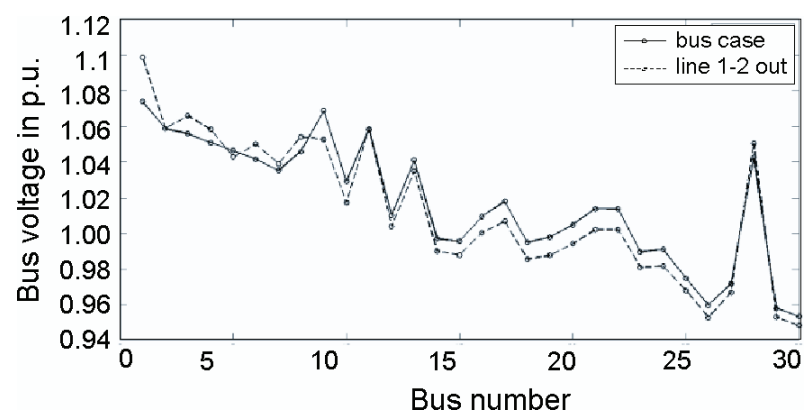

deviation. Total number of generations $=300$, population size $=60$ and number of runs $=20$ for all these observations. From Figure 8 and Figure 18 HGO exhibits quick convergence characteristics. HGO outperforms DE and standard PSO algorithm due to additional searching abilities. 


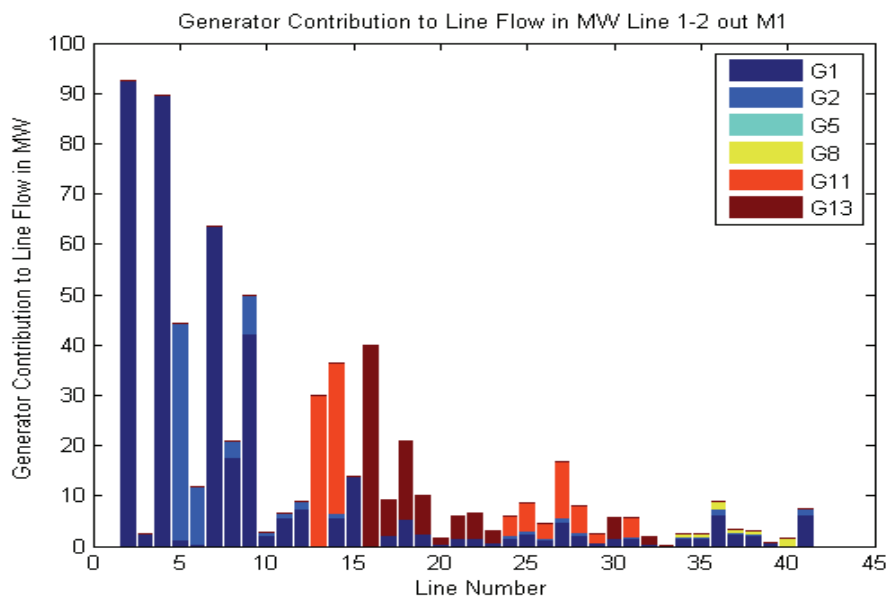

Fig. 13. Generator contributions to line flow at base case (Case III)

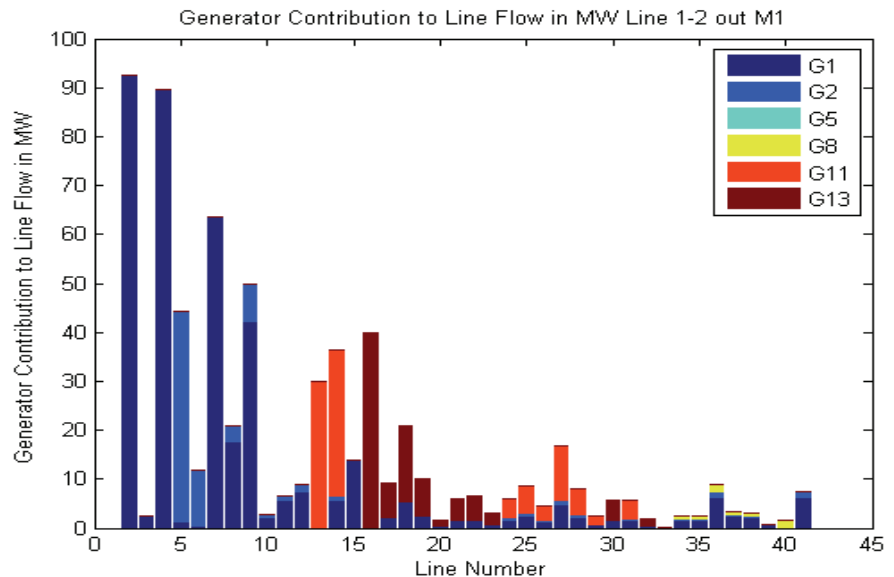

Fig.14. Generator contributions to line flow at line 1-2 out (Case III)

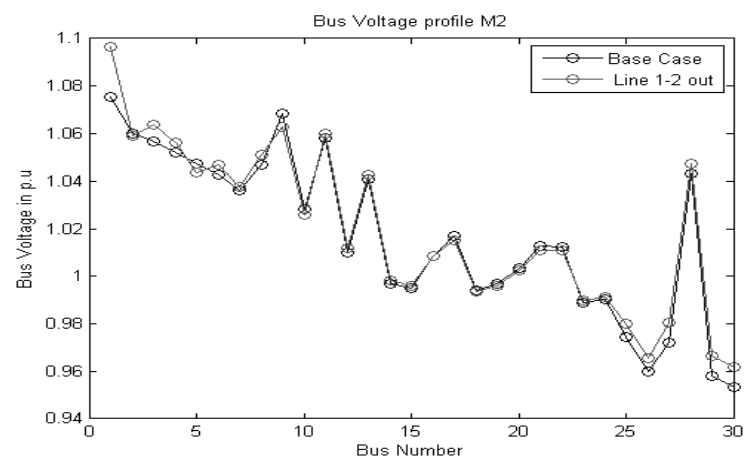

Fig. 15. Bus Voltage Profile (Case IV) 
Fig. 16. Generator contributions to line flow at base case (Case IV)

Fig. 17. Generator contributions to line flow at line 1-2 out (C- IV)
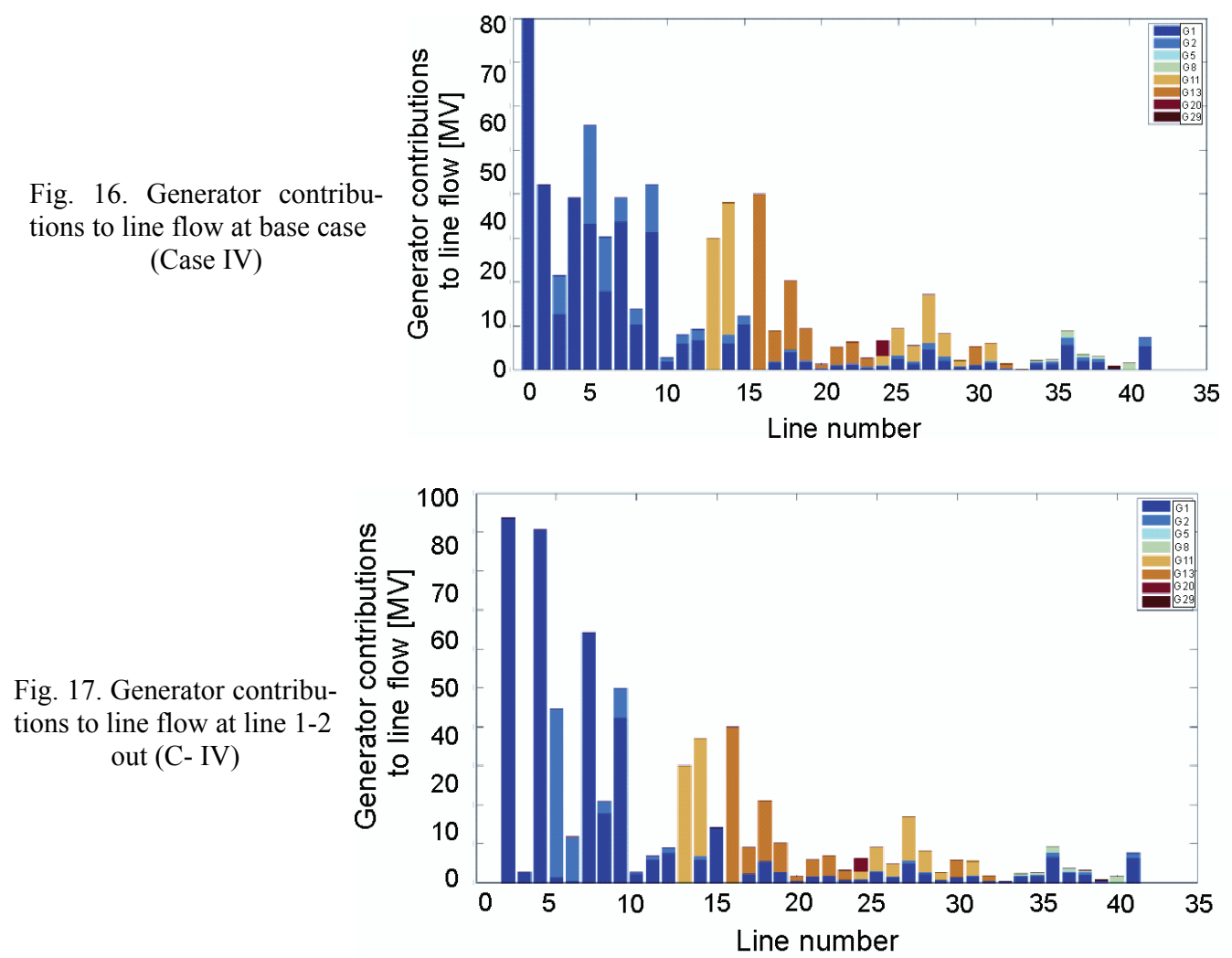

Fig. 18. Convergence characteristics (Case IV)

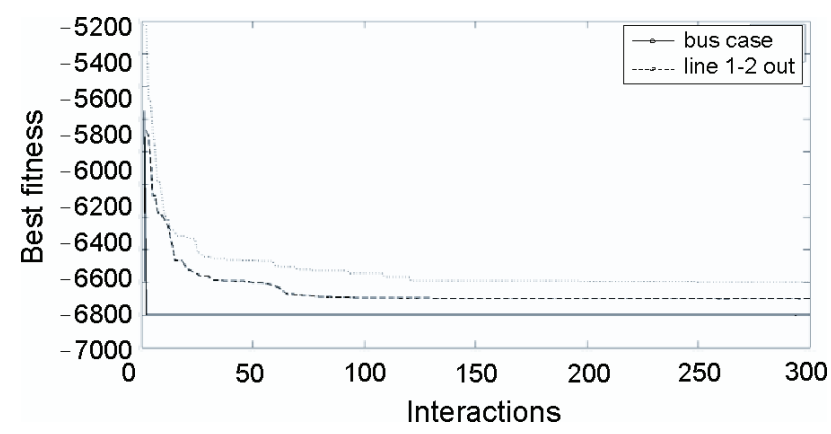

\section{Conclusion}

In this paper a new optimization problem is formed, which includes power system security, optimal power flow, transmission cost under bilateral, multilateral transactions. This optimization problem finds the solution to minimize the generation and transmission cost at the fixed load condition and also to enhance security at pre and post contingency periods. Apart from generators available in the standard IEEE system, bilateral and multilateral transactions also 
considered. Cost of bilateral and multilateral transactions are not included in the cost function. The effectiveness of the HGO algorithm to find the solution for the mentioned problem is studied for IEEE 30 bus with 4 cases. Optimization results produced by HGO are compared with DE and PSO. From the statistical analysis it is found that HGO performs well. From the convergence characteristic, HGO took a less number of iterations to find optimum value. Optimal placement of IPP's in deregulated environment is addressed well by comparing different transactions and their associated costs in IEEE 30 bus system. Comparing other Security constrained OPF this paper addresses the expected security cost in the post contingency period. Power flow tracing is applied to find the contribution of generators and IPPs to the line flow. New evolutionary algorithm HGO is applied to Indian utility -62 bus system to find its effectiveness. Other algorithms for this problem fail to converge. The result is not displayed.

\section{References}

[1] Alsac O., Stott B., Optimal load flow with steady state security. IEEE Transactions on Power Apparatus and Systems 93: 745-751 (1974).

[2] Aguado J. A., Quintana V.H.., Optimal power flows of interconnected power systems. IEEE Power Engineering Society Summer Meeting 2: 814-819 (2009).

[3] Padhy N.P., Kumari L., Evolutionary Programming Based Economic Power Dispatch Solutions With Independent Power Producers. 2004 IEEE International Conference on Electric Utility Deregulation, Restructuring and Power Technologies (DRPT2004) Hong Kong 1: 172-177 (2004).

[4] Kumar A., Chanana S., Security Constrained Economic Dispatch with Secure Bilateral Transactions in Hybrid Electricity Markets. Power System Technology and IEEE Power India Conference, pp. 1-6 (2008).

[5] Cheng J.W.M., McGills D.T., Galiana F.D., Probabilistic security analysis of bilateral transactions in a deregulated environment. IEEE Transaction on Power systems 14(3): 1153-1159 (1999).

[6] Raglend J., Karthikeyan P., Kothari D.P., Comparison of Intelligent Techniques to Solve Economic Load Dispatch with Bilateral and Multilateral Transactions. TENCON 2008 IEEE Region 10 Conference, Hyderabad, pp. 1-6, 19-21(2008).

[7] Allen Wood J., Wollenberg B.F., Power Generation Operation and Control. Wiley (1996).

[8] Udupa A.N., Purushothama G.K., Parthasarathy K., Thukaram D., A fuzzy control for network overload alleviation. Electr. Power Energy Syst. 23: 119-129 (2001).

[9] Laurent Lenoir, Innocent Kamwa and Louis-A. Dessaint., Overload Alleviation with PreventiveCorrective Static Security Using Fuzzy Logic. IEEE Trans. Power Syst. 24(1): 134-145 (2009).

[10] Devaraj, Yegnanarayana B., Genetic-algorithm-based optimal power flow for security enhancement. IEE Proc.-Gener. Transm. Distrib. 152(6): 899-905 (2005).

[11] Shahidehpour M., Yamin H., Li H., Market Operation in Electric Power Systems. John Wiley \& Sons Inc., New York, USA (2002).

[12] Bialek J.W., Tracing the flow of electricity. IEE Proceedings Generation Transmission and Distribution 143 (July (4)) 313-320 (1996).

[13] Kirschen D., Allan R., Strbac.G., Contribution of individual generations to loads and flows. IEEE Transactions on Power Systems 12 (February (1)) 52-60 (1997).

[14] Bialek J.W., Kattuman., Real and reactive power tracking: Proof of concept and feasibility study. Electric Power Research Institute, Tech. Rep. TR112416 (1999).

[15] Pablo Onate, Juan M. Ramirez, Carlos A. Coello Coello., An optimal power flow plus transmission costs solution. Electric Power Systems Research 79(8): 1240-1246 (2009).

[16] Lai L.L., Yokohoma R., Zhao M., Improved Genetic Algorithm for Optimal Power Flow Under Both Normal and Contingent Operation States. International journal on Electrical Power Energy System 19: 287-291 (1997). 
[17] Abido M.A., Optimal Power Flow Using Particle Swarm Optimization. International journal on Electrical Power and Energy Systems 17(2): 563-571 (2002).

[18] Dai, Zhu, Chen., Seeker optimization algorithm. [in:] Computational Intelligence and Security 4456, Lecture Notes in Artificial Intelligence, Y. Wang, Y. Cheung, H. Liu (Eds.) Berlin, Germany: Springer-Verlag, pp. 167-176 (2007).

[19] Chaohua Dai, Weirong Chen, Yunfang Zhu., Seeker optimization algorithm for digital IIR filter design. IEEE Transactions on Industrial Electronics 57(5): 1710-1718 (2009).

[20] Chaohua Dai, Weirong Chen, Yunfang Zhu, Xuexia Zhang., Seeker optimization algorithm for optimal reactive power dispatch. IEEE Transactions on Power Systems 24(3): 1218-1231 (2009).

[21] Chaohua Dai, Weirong Chen, Yunfang Zhu, Xuexia Zhang., Reactive power dispatch considering voltage stability with seeker optimization algorithm Electric Power System Research 79(10): 14621471 (2009).

[22] Chaohua Dai, Weirong Chen, Lixiang Li et al., Seeker optimization algorithm for parameter estimation of time-delay chaotic systems. Physical Review E, 83, 036203 (2011).

[23] Chaohua Dai, Weirong Chen, Lili Ran, Yi Zhang, Yu Du., Human Group Optimizer with Local Search. Lecture Notes in Computer Science 6728: 310-320 (2011).

[24] Condren J,. Gendra T.W., Damrongkulkamjorn P., Optimal power flow with expected security costs. IEEE Transactions on Power System 21(2): 541-547 (2006).

[25] Zimmerman R., Gan D., MATPOWER: A Matlab power system simulation package, Software package available at ttp://www.pserc.cornell.edu/matpower

[26] Gnanadass R., Pathey N.P., Manivannan., Assessment of available transfer capability for practical power systems with combined economic emission dispatch. Electric Power Systems Research 69: 267-276 (2004). 